\title{
The World Incidence and Prevalence of Autoimmune Diseases is Increasing
}

\author{
Aaron Lerner ${ }^{1, *}$, Patricia Jeremias ${ }^{2}$, Torsten Matthias ${ }^{2}$ \\ ${ }^{1}$ B. Rappaport School of Medicine, Technion-Israel Institute of Technology, Haifa, Israel \\ ${ }^{2}$ AESKU.KIPP Institute, Wendelsheim, Germany \\ *Corresponding author: aaronlerner1948@gmail.com
}

Received September 27, 2015; Revised October 29, 2015; Accepted November 11, 2015

\begin{abstract}
Epidemiological data provide evidence of a steady rise in autoimmune disease throughout Westernized societies over the last decades. Multiple publications exist, describing past or actual incidences/prevalence of individual autoimmune diseases, however, long term studies on selected populations are scarce. Aims: to calculate the \% increases per year of autoimmune diseases frequencies worldwide, analyze the differential increases per country and disease, and identify geoepidemiological trends. Methods: A systematic review was performed to identify incidence and prevalence of autoimmune diseases. 30 Studies from the last 30 years were identified using Medline, Google, and Cochrane Library databases. Only long-term regional or national follow-ups are reported. Results: The means \pm s.d. of the net \% increased /year incidence and prevalence of autoimmune diseases worldwide were 19.1 \pm 43.1 and 12.5 \pm 7.9 , respectively. Rheumatic, endocrinological, gastrointestinal and neurological autoimmune diseases revealed the following annual \% increases per year: 7.1, 6.3, 6.2, and 3.7, respectively. In all of these, differences between old vs new frequencies were highly significant $(p<0.0001)$. Comparing various autoimmune diseases, celiac disease increased the most and the highest increase in incidence, comparing old to new surveys is allocated to myasthenia gravis. Despite considerable variations between the countries, celiac, type 1 diabetes and myasthenia gravis frequencies increased the most in Canada, Israel and Denmark, respectively. Frequencies of the autoimmune diseases increased significantly in the West and North when compared to East and South, respectively. Conclusions: Despite multiple reports on autoimmune diseases frequencies, long-term longitudinal follow-ups are scarce. Incidences and prevalences have increased significantly over the last 30 years. Rheumatic, endocrinological and gastrointestinal autoimmune diseases in Israel, Netherlands, USA and Sweden increased the most. These observations point to a stronger influence of environmental factors as opposed to genetic factors on autoimmune disease development.
\end{abstract}

Keywords: autoimmune disease, incidence, prevalence, surge, geoepidemiology

Cite This Article: Aaron Lerner, Patricia Jeremias, and Torsten Matthias, "The World Incidence and Prevalence of Autoimmune Diseases is Increasing.” International Journal of Celiac Disease, vol. 3, no. 4 (2015): 151-155. doi: 10.12691/ijcd-3-4-8.

\section{Introduction}

Increasing evidence is accumulating for a steady rise in the frequency of autoimmune diseases $(\mathrm{AD})$, in the last decades [1]. In fact, the rise in ADs parallels the surge in allergic and cancer conditions while infections are less frequent in the Western societies, creating the basis for the hygiene hypothesis [2]. Multiple sclerosis (MS), type 1 diabetes (IDDM), inflammatory bowel diseases (mainly Crohn's disease) (IBD), systemic lupus erythematosus (SLE), primary biliary cirrhosis, myasthenia gravis (MS), autoimmune thyroiditis (AT), hepatitis and rheumatic diseases (RA), bullous pemphigoid, and celiac disease (CD) are several examples [3,4]. Their relationship to socioeconomic status, their rapid increase in developed countries and observations in selected migrant populations, indicate some form of environmental impact, rather than long-term genetic influences which are driving these recent evolutionary processes [1,2,3,4,5]. Among many others, three major environmental factors, strongly related to socioeconomical status are suspected to drive these phenomena: infections, ecology and nutrition. The purpose of the present review is to calculate the surge per year of $\mathrm{AD}$ frequencies worldwide and analyze the differential increases of $\mathrm{AD}$ per country and disease identifying geoepidemiological trends. There is a plethora of publications on the incidences/prevalences of ADs in medical literature, most of them describe past or actual frequencies of individual ADs, however, long term comparative follow-up studies on selected populations, in individual countries are scarce.

\section{Methods}

A Medline search was performed using the following search words: autoimmune disease or syndrome, incidence, prevalence or frequency, spanning the period 1985-2015. 
Special emphasis was given to the identification of increase/surge or decrease in incidence/prevalence worldwide. Suitable publications were identified by periodical scans of PubMed but also Google, and the Cochrane Library databases, were screened. Original papers, in the English language, especially those on epidemiology of autoimmune diseases commonly encountered, were identified. Foreign language papers with English abstracts were also identified. Only longterm regional or national longitudinal follow-ups are reported. Hospital archived data, specific high-risk groups or case reports, were excluded. Entities were validated and used when directly stating at least one of the abovementioned epidemiological indices. Chi-square test or Fisher`s exact test were used for categorical data and pvalues less than 0.05 were considered as statistically significant. Statistical analyses were performed using the statistics software MedCalc version 15.6.1.

\section{Results}

30 studies were identified. The means \pm s.d. of the net \% increased /year incidence and prevalence of ADs worldwide were $19.1 \pm 43.1$ and $12.5 \pm 7.9$, respectively (Figure 1). Of interest, grouping the different ADs to disease categories, the highest net \% increase per year was noted in the rheumatologic (7.1), followed by endocrine (6.3), gastrointestinal (6.2) and neurological diseases (3.7). In all of these, differences between old vs new frequencies were highly significant $(\mathrm{p}<0.0001)$ (Figure 2$)$. The table inserted in Figure 2 details the diseases and the countries included in the 4 disease categories.

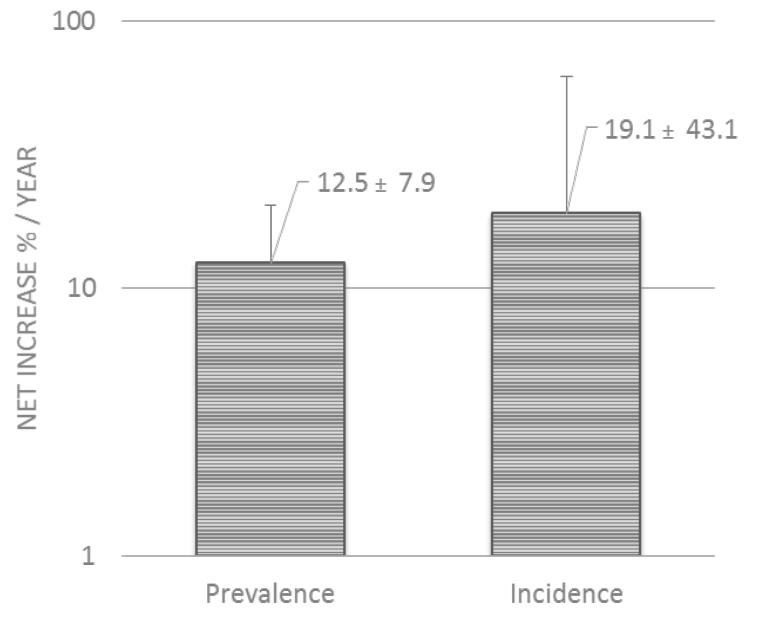

Figure 1. The net \% increase/year of the incidence and prevalence of autoimmune diseases worldwide

(A) 100

(B)

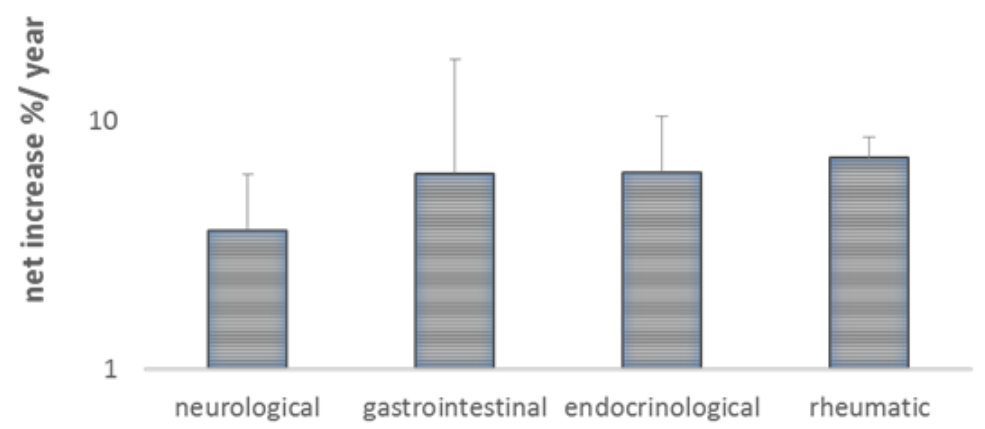

disease groups

\begin{tabular}{|c|c|c|c|c|}
\hline disease kind & $\begin{array}{l}\text { statistical } \\
\text { significance ( } p \text {; } \\
\text { old vs. new) }\end{array}$ & $\begin{array}{c}\text { Mean net } \\
\text { increase \% } \\
\text { /year }\end{array}$ & countries & diseases \\
\hline neurological & $<0.0001$ & $3.7 \pm 2.5$ & Finland, Denmark, Norway, Italy, Spain & MS, Myasthenia Gravis \\
\hline gastrointestinal & $<0.0001$ & $6.2 \pm 11.5$ & $\begin{array}{c}\text { Denmark, Canada, Sweden, USA, Finland, Israel, } \\
\text { Netherlands, UK, Czech, Scotland, Spain, Estonia, New } \\
\text { Zealand }\end{array}$ & Autoimmune Hepatitis, IBD, Chron's, Celiac Disease \\
\hline endocrinological & 0.02 & $6.3 \pm 4.2$ & Brazil, Canada, Israel, Serbia, Europe & Autoimmune thyroiditis, IDDM \\
\hline rheumatic & 0.02 & $7.14 \pm 1.5$ & Canada, UK & SARD, RA, SLE \\
\hline
\end{tabular}

Figure 2. (A) The net \%/year increases of diseases' categories. (B) The table below is detailing the different diseases and countries surveyed

The net increase/year of various diseases in specific countries and old vs. new surveys of incidence/prevalence of various autoimmune diseases, are shown in Figure 3A and $3 B$, respectively. Figure $3 \mathrm{~A}$ shows the total net increase of Old vs. New surveys of incidence/prevalence of various autoimmune diseases, (mean time interval RA 14.5, AT 13.5, Chron`s 11.5, IBD 11.0, IDDM 17.8, Celiac 16.8 and MG 27 years, respectively), During the reported time interval. $C D$ increased the most (26.3/year \%) and the higher increase in incidence, comparing old to new surveys is allocated to MG (432\%)
(Figure 3A and Figure 3B). Geoepidemiologically, the following countries had high to low \% increases /year of AD frequencies: Israel, Netherlands, USA, Sweden, UK, Finland, Canada, and Denmark with 12.9, 10.0, 8.8, 8.4, 7.8, 7.6, 7.3 and $6.3 \%$, respectively. There was no statistically significant difference between children and adults in the increase of $\mathrm{AD}$ incidence/ prevalence $(\mathrm{p}=0.8036)$. Figure 4 shows, as an example, two frequent ADs like CD and IDDM and one, less frequent one like MG surveys in various countries. A considerable variation is noticed between the countries. CD, IDDM and MG 
frequencies increased the most in Canada, Israel and Denmark, respectively.

$\square$ new incidence / prevalence $\quad$ in old incidence / prevalence

(A)

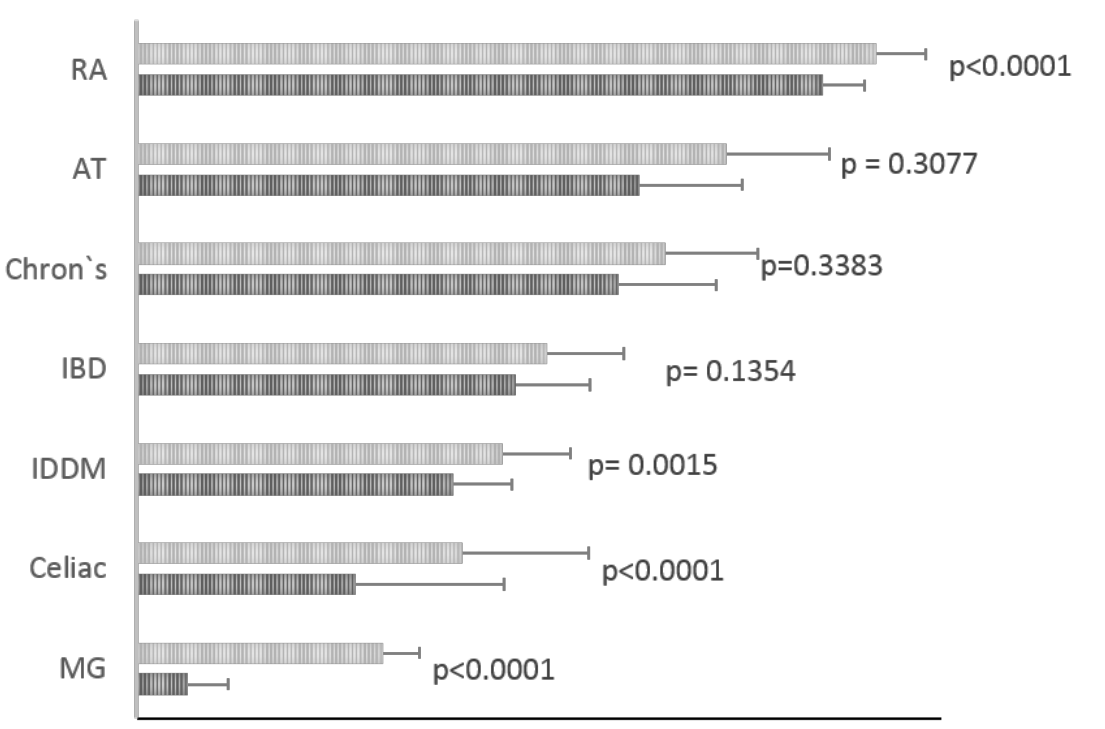

(B)

\begin{tabular}{|c|c|c|}
\hline Disease & Countries & Survey \\
\hline RA & Canada & $1995-2010$ \\
\hline AT & Brazil, Denmark & $1992-2012$ \\
\hline Chron's & Sweden, USA & $1991-2007$ \\
\hline IBD & Canada & $1994-2005$ \\
\hline IDDM & Canada, Israel, Serbia, Europe & $1982-2010$ \\
\hline Celiac & $\begin{array}{l}\text { Canada, Denmark, Finland, Israel, } \\
\text { Netherlands, USA, Sweden, UK, } \\
\text { Czech Republic, Scotland, Spain, } \\
\text { Estonia, New Zealand }\end{array}$ & $1950-2011$ \\
\hline MG & Denmark, Norway, Italy, Japan & 1950-2006 \\
\hline
\end{tabular}

Figure 3. (A) Old vs. New surveys of incidence/prevalence of various autoimmune diseases. (B) The list of various diseases in specific countries and the years' ranges

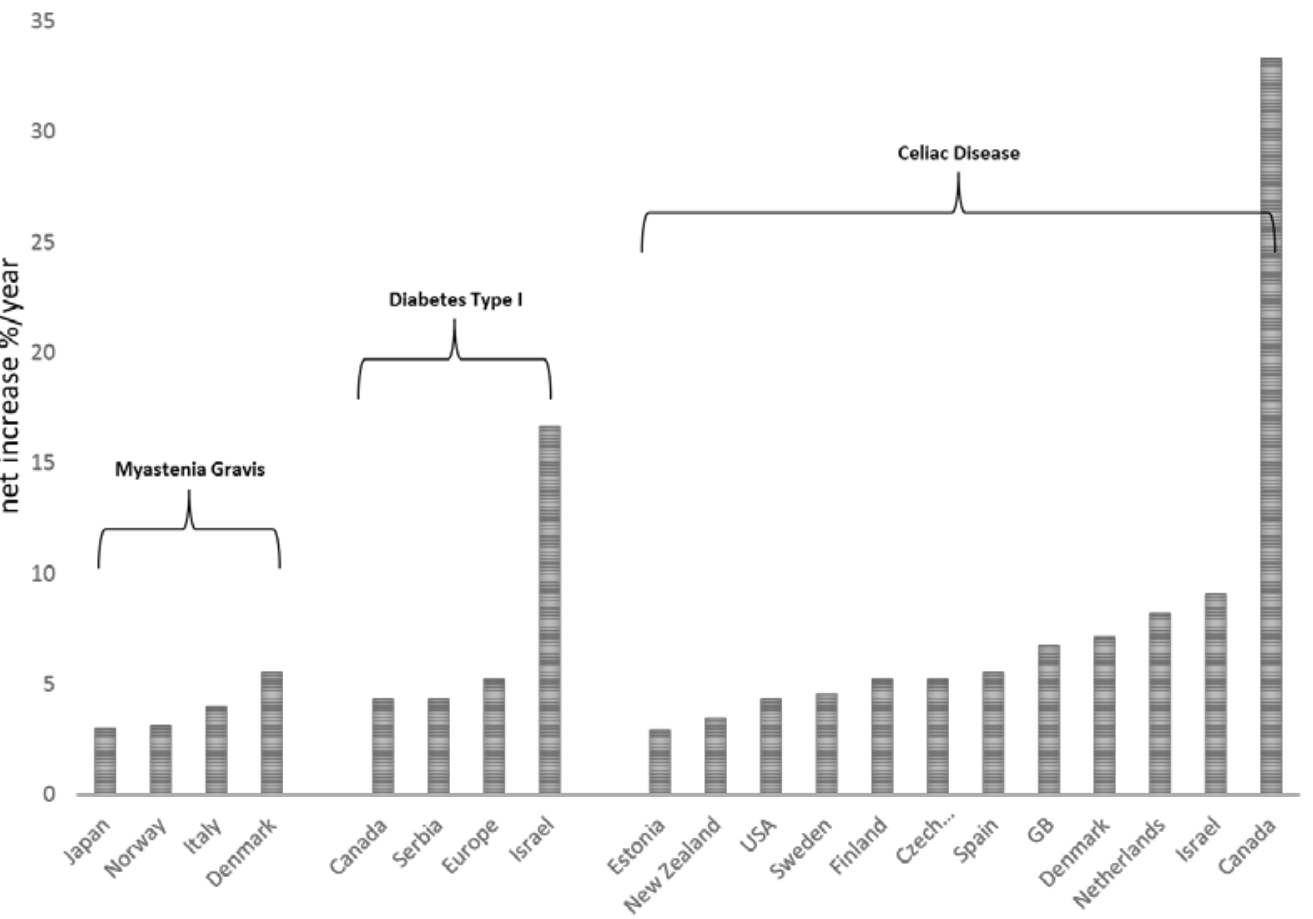

Figure 4. The net increase \%/year of 3 autoimmune diseases in the surveyed countries 
The geoepidemiologic trend of the net increase \%/year of the various ADs is described in Figure 5. Frequencies of the ADs increased significantly in the West and North when compared to East and South, respectively.
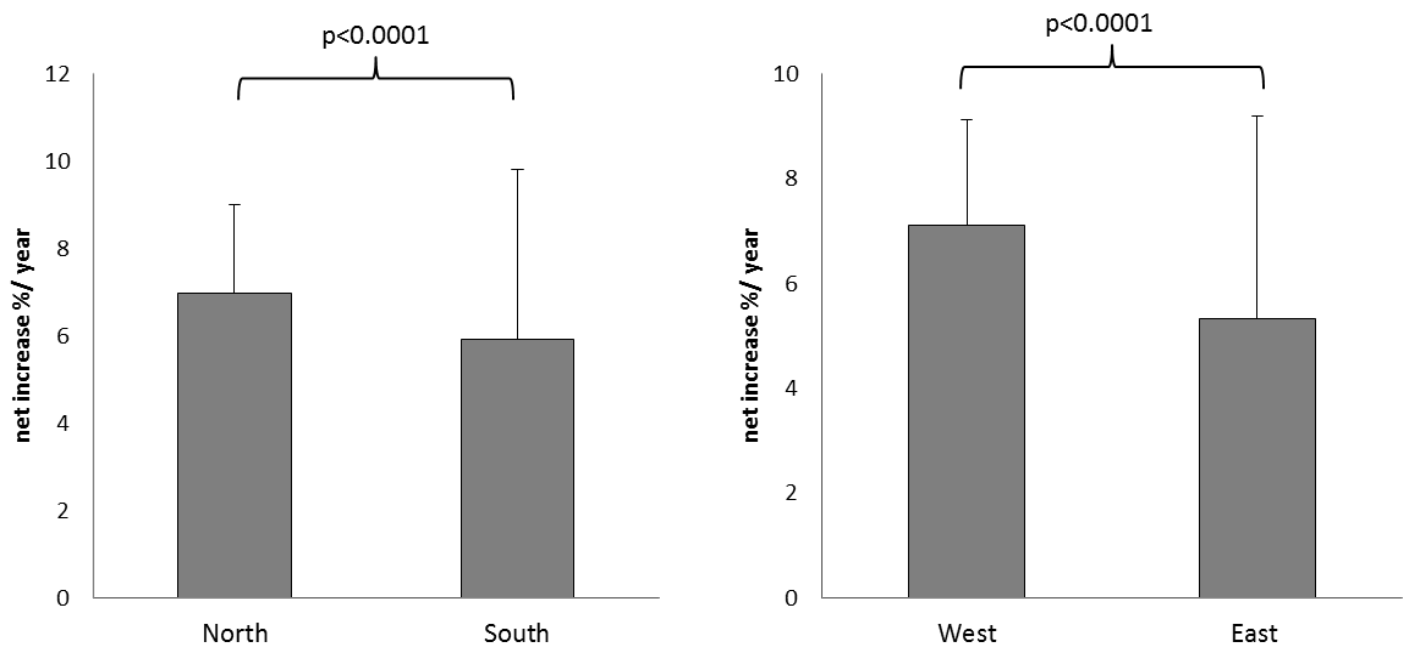

Figure 5. The geoepidemiology of the net increase \%/year of autoimmune diseases

\section{Discussion}

Population-based estimates of the incidence/prevalence of ADs in different countries are crucial for investigating possible etiologies or influencing environmental factors or underlining risk factors.

Quantification of the likely healthcare burden and the planning of future strategies to face the ongoing epidemic of ADs is of no less importance. More so, the beneficial aspects of early recognition of ADs are well known. Multiple AD related complications can be prevented or treated when the disease is diagnosed early enough. The increased awareness responsible for the surge in diagnosed patients, needs to continue together with a lower threshold for screening high risk populations using a cheap, non-invasive and reliable antibody biomarker to prevent delays in diagnosing such a common but preventable disease $[6,7,8]$.

The net increased incidences/prevalences of ADs in the Northern and Western countries, compared to the Southern and Eastern ones follows the global geoepidemiological trends of autoimmune diseases $[9,10]$. By reviewing available literature, it can be deduced that frequency of ADs have increased significantly over the last 30 years. The recent outbreak of autoimmune diseases in industrialized countries has brought into question the factors contributing to this increased incidence. Given the constancy of genetics, growing attention has focused on environmental factors, and in particular, the western lifestyle [3,4,11]. Indeed, over the last few decades significant changes in western dietary habits, environmental surroundings and pollution exposure, infectious habitat and stress load, have led to a parallel rise in autoimmune diseases. Thus, ADs occupies an important place in this environmental mosaic of autoimmunity, allergy and cancer surges [2].

The present study can serve as a platform for geoepidemiological inquiries, concerning offending/ defending environmental changes. If $\mathrm{CD}$ is taken as an example, how comes the surge in the disease frequency was much higher in Canada, Israel and Netherlands, compared to the low rates in Estonia and New Zealand?
Considering the diseases, what environmental forces contributed to the higher surge of CD and MG, compared to IBD and IDDM? Comparing the disease categories, why are rheumatic disease surges higher than the neurological ones? Is it the infectious or stress loads? The intestinal disbiosis allocated to specific diseases? Driving openers of the intestinal tight-junctions? Or is it the increased public/professional awareness or the improved diagnosis? A vast list of questions and so few answers.

The present study has multiple biases and drawbacks. The follow up surveys were not done by the same team nor by the same methodology, some of the relevant studies might have escaped detection, the genetic makeup and the environment are different in the screened countries, the environmental forces are dynamic and most probably changed during the last decades, the actual public and professional awareness to autoimmunity and the diagnostic bio-markers have evolved tremendously.

Despite those biases, the present study highlights the significant surge in AD incidence and prevalence, detailing the variations in disease entities and the corresponding countries and substantiate the geoepidemiological trends. The present literature survey is not aiming to investigate etiologies or environmental factors affecting autoimmune induction or progression. It is expected that an improved knowledge of the worldwide distribution of autoimmune disorders will help to understand the role of different genetic factors and different environmental influences involved in autoimmunogenesis. At a public level the epidemiological studies are necessary to assess the social and economic burdens impacting the health systems in the different countries, and worldwide.

\section{References}

[1] Lohi S, Mustalahti K, Kaukinen K, Laurila K, Collin P, Rissanen $\mathrm{H}$ et al. Increasing prevalence of coeliac disease over time. Aliment Pharmacol Ther. 2007;26:1217-1225.

[2] Bach JF. The effect of infections on susceptibility to autoimmune and allergic diseases. N Engl J Med 2002; 347: 911-920.

[3] Lerner A, Matthias T. Changes in intestinal tight junction permeability associated with industrial food additives explain the rising incidence of autoimmune disease. Autoimmun Rev. 2015;14:479-489 
[4] Lerner A, Matthias T. Possible association between celiac disease and bacterial transglutaminase in food processing: a hypothesis. Nutr Rev. 2015;73:544-552.

[5] Lerner A. The last two millennias eco-catastrophes are the driving forces for the potential genetic advantage mechanisms in celiac disease. Med Hypotheses. 2011, 77;773-776.

[6] Ludvigsson JF, Rubio-Tapia A, van Dyke CT, et al. Increasing incidence of celiac disease in a North American population. Am J Gastroenterol. 2013;108:818-824.

[7] Lerner A, Matthias T. Increased knowledge and awareness of celiac disease will benefit the elderly. Intern. J of Celiac Dis. 2015;3:112-114.
[8] Lerner A, Neidhöfer S, Matthias T. Serological markers and/or intestinal biopsies in the case-finding of celiac disease. Editorial, Internat. J Celiac dis. 2015;3:53-55.

[9] Agmon-Levin N, Lian Z, Shoenfeld Y. Explosion of autoimmune diseases and the mosaic of old and novel factors. Cell Mol Immunol. 2011;8:189-192.

[10] Ramos-Casals M, Brito-Zerón P, Kostov B, et al. Google-driven search for big data in autoimmune geoepidemiology: analysis of 394,827 patients with systemic autoimmune diseases. Autoimmun Rev. 2015;14:670-679.

[11] Manzel A, Muller DN, Hafler DA, Erdman SE, Linker RA, Kleinewietfeld M. Role of "Western diet" in inflammatory autoimmune diseases. Curr Allergy Asthma Rep. 2014;14:404. 